\title{
D-01
}

\section{ESTRATEGIAS PARA REDUCIR EL COSTE ENERGÉTICO EN COMUNIDADES DE REGANTES}

\author{
$\underline{\text { Yusta Loyo, J. M. (1) }}$ \\ ${ }^{1}$ Dr. Ingeniero Industrial, Profesor Titular, Universidad de Zaragoza, C/María de Luna 3 \\ 50018 Zaragoza, jmyusta@unizar.es
}

\section{Resumen}

Los costes de suministro eléctrico de las comunidades de regantes se han duplicado en los últimos años desde la desaparición de la tarifa especial de riego agrícola en 2008. Aunque el mercado eléctrico liberalizado ofrece oportunidades para elegir al suministrador de energía y negociar los precios, las comunidades se han visto gravemente penalizadas por el incremento del término fijo de potencia contratada, a pesar del esfuerzo por concentrar al máximo el consumo de energía en los periodos más baratos. La Comunidad General de Riegos del Alto Aragón ha desarrollado distintas iniciativas desde 2010 para reducir el coste energético de las comunidades de regantes, entre otras el diseño de una plataforma online para el seguimiento de consumos y facturas eléctricas, el desarrollo de herramientas para la programación horaria del bombeo, el ajuste óptimo de las potencias contratadas, la negociación colectiva de los contratos de suministro o el estudio permanente de alternativas para la reducción del coste mediante fuentes de energías alternativas.

\section{1- Introducción}

El sistema de Riegos del Alto Aragón (RAA) comprende 52 comunidades de regantes con una superficie en regadío superior a 135.000 hectáreas, que utilizan anualmente 50 millones de kilovatios-hora de energía eléctrica en 42 estaciones de bombeo de agua.

En el sistema de RAA y en gran parte de los regadíos españoles, las inversiones para su modernización se realizaron teniendo en cuenta en los estudios previos de rentabilidad económica un coste del suministro eléctrico para el bombeo de agua que a día de hoy se ha duplicado. Hasta 2008, las tarifas especiales de riego agrícola garantizaban unos precios regulados por parte del gobierno para el suministro de energía a las instalaciones de bombeo de las comunidades de regantes, pero desde esta fecha las comunidades tienen que contratar la electricidad en el mercado liberalizado a precios muy superiores, lo que pone en serio riesgo de supervivencia muchas de las instalaciones modernizadas, al tener que hacer frente a precios de la electricidad caros y además volátiles e inciertos a lo largo de los meses del año. Frente a un precio medio de 7,7 céntimos de euro por kWh en 2007, las comunidades de regantes del sistema de RAA han tenido que hacer frente a precios superiores a 15 céntimos de euro por kWh en 2014, IVA incluido.

En el aumento del coste del suministro eléctrico ha influido especialmente el incremento de peajes de acceso a la red, la parte del precio de la electricidad que pagan los consumidores en el mercado libre y que utiliza el gobierno para recaudar los costes regulados, que no ha dejado de crecer exponencialmente en los últimos años. Sólo el incremento del término de potencia de estos peajes en un $115 \%$ en virtud de la orden ministerial IET/1491/2013 supuso un incremento medio desde el 3 de agosto de 2013 del $20 \%$ del coste final de la electricidad en las comunidades de regantes del sistema de Riegos 
del Alto Aragón, que han visto además como este término fijo que depende de la potencia contratada supone más del $40 \%$ del precio final de la electricidad. Este porcentaje es mucho más elevado en los bombeos de las comunidades de regantes que en otros perfiles de consumidores por motivo de la singular estacionalidad del consumo de electricidad en los meses de campaña de riego, concentrada entre los meses de mayo a septiembre en el caso de Riegos del Alto Aragón.

A pesar del esfuerzo por concentrar al máximo el consumo de energía en los periodos más baratos (especialmente el periodo P6 que comprende las noches, los fines de semana y todo el mes de agosto) y consecuentemente reducir la potencia contratada en los periodos más caros renunciando en algunos casos a su utilización, la obligación de contratar la potencia durante los doce meses del año grava injustamente la factura eléctrica de los regantes. Las comunidades de regantes de RAA concentran en promedio el $80 \%$ de su consumo en el periodo P6, periodo valle del sistema eléctrico, coadyuvando a la mejora del factor de carga del sistema eléctrico y a la utilización más eficiente de las infraestructuras eléctricas de generación, transporte y distribución, y sin embargo son penalizadas en el coste del suministro eléctrico.

El único alivio para el sector procede de la aprobación de la reducción parcial del $85 \%$ del impuesto sobre la electricidad para riegos agrícolas desde el 1 de enero de 2015. Ha supuesto una reducción del coste mensual del suministro eléctrico del $4,35 \%$. A esto se suma la aprobación mediante el Real Decreto-Ley 9/2015 y la Orden IET/2735/2015 de la reducción de los pagos de capacidad, que sin embargo apenas tiene impacto en las comunidades de regantes, ya que no se aplica este concepto de coste en el periodo P6, periodo horario en el que se realiza el $80 \%$ del consumo eléctrico en el regadío.

\section{2 - Iniciativas para la reducción de costes energéticos}

Para contrarrestar la situación descrita en el apartado anterior, la Comunidad General de Riegos del Alto Aragón viene desarrollando distintas iniciativas desde 2010 para reducir el coste energético de las comunidades de regantes en el mercado eléctrico liberalizado, que se presentan brevemente a continuación.

\subsection{Sistema online de seguimiento de facturas y consumos eléctricos}

Desde 2010 se mantiene un sistema de seguimiento anual de consumos eléctricos y revisión de las facturas, para garantizar el correcto pago del servicio eléctrico, revisar las posibles desviaciones de coste de cada comunidad de regantes y recoger centralizadamente toda la información del consumo de las comunidades. Esto permite desarrollar posteriormente estudios y análisis para optimizar los parámetros de la contratación y reducir el coste del suministro.

\subsection{Programación del bombeo en horas más baratas}

Las comunidades de regantes planifican habitualmente el funcionamiento de las bombas para maximizar su uso en las horas con electricidad más barata, en función del calendario anual de periodos tarifarios de cada tarifa de acceso. Para contribuir a esta tarea se han desarrollado modelos matemáticos de previsión de consumos diarios de energía en función de las peticiones de agua y se han propuesto herramientas informáticas para la programación horaria del bombeo con objeto de minimizar el coste del suministro eléctrico.

\subsection{Ajuste óptimo de potencias contratadas}


Dado el elevado coste del término fijo de potencia, al comienzo de cada campaña de riego se propone la revisión de las potencias contratadas. Estas acciones han permitido reducir el coste un promedio del $10 \%$ en los últimos años.

Para ajustar las potencias contratadas en cada estación de bombeo, se procede mediante el siguiente procedimiento:

- Análisis de las necesidades de demanda de potencia de bombeo en función de la planificación de cultivos y reservas hídricas disponibles para la campaña.

- Cálculo de potencias óptimas en cada periodo tarifario incluso incurriendo en excesos de potencia en los meses de verano.

- Elección de potencia a contratar: en suministros en alta tensión han de ser crecientes en los distintos periodos ( $P 1 \leq P 2 \leq P 3 \leq P 4 \leq P 5 \leq P 6)$, estando limitada la modificación de la potencia en el periodo 6 por valores de potencia máxima y mínima en función de los transformadores de medida del centro de transformación.

- Comunicación por escrito a la empresa comercializadora de las potencias a contratar.

En la Figura 1 se muestra un ejemplo real de optimización de potencias contratadas donde se observa que aun incurriendo en excesos de potencia se obtiene un notable ahorro por la disminución del coste fijo anual de potencia en los periodos P1 a P5. No se modifica la potencia del periodo P6 para no perder derechos de acometida y no tener que realizar cambios en los equipos de medida.

En este ajuste es preciso observar tres precauciones:

- Según el artículo 5 del Real Decreto 1164/2001 no se pueden realizar modificaciones de potencia hasta transcurridos doce meses de la anterior (algunas distribuidoras sí permiten aumentos sucesivos de potencia en un plazo inferior a un año pero no reducción).

- Los aumentos de potencia pueden suponer costes por derechos de acometida, las reducciones sólo derechos de actuación en el equipo de medida.

- La nueva regulación de los derechos de acometida del Real Decreto 1048/2013 permite conservar los derechos de extensión (en alta tensión durante 5 años) pero no los derechos de acceso. Si se reduce la potencia contratada en el periodo 6 y de nuevo se amplía en un plazo no superior a 5 años, se abonarán de nuevo derechos de acceso. Si ha transcurrido un plazo superior a 5 años, se abonarán derechos de acceso y derechos de extensión.

La figura legal del contrato de temporada no se aplica en ninguna comunidad de regantes de este sistema. Aunque de escasa utilización previa, el contrato de temporada de cinco meses regulado mediante el Real Decreto 1578/2008 estaba siendo firmemente valorado como una alternativa para reducir el coste del suministro en conjunto con otras fuentes de energía eléctrica autónomas en los 7 meses restantes, ya que permitía el pago del término de potencia con pequeñas penalizaciones del $35 \%$ en los meses de junio y julio y del $15 \%$ en el resto de meses de la temporada de riego comprendidos en los cinco meses del contrato, pero evitaba el pago del término fijo de potencia el resto del año. Dado que el Real Decreto 1578/2008 ha sido derogado, queda únicamente vigente la figura del contrato de temporada regulada por el real decreto 1164/2001, claramente inviable porque penaliza el término de potencia en un $100 \%$ en los meses de junio y julio y en un $50 \%$ en el resto. 


\begin{tabular}{|c|c|c|c|c|c|c|}
\hline $\begin{array}{c}\text { POTENCIAS } \\
\text { CONTRATADAS }\end{array}$ & Periodo 1 & Periodo 2 & Periodo 3 & Periodo 4 & Periodo 5 & Periodo 6 \\
\hline Iniciales & 12 & $\mathbf{k W}$ & $\mathbf{k W}$ & $\mathbf{k W}$ & $\mathbf{k W}$ & $\mathbf{k W}$ \\
\hline Modificadas & $\mathbf{1 2}$ & 1382 & 1382 & 1382 & 1382 & 1882 \\
\hline
\end{tabular}

\begin{tabular}{|c|c|c|}
\hline Resumen costes AÑO 2015 & $\begin{array}{c}\text { Con nuevas } \\
\text { potencias }\end{array}$ & $\begin{array}{c}\text { Con } \\
\text { potencias } \\
\text { anteriores }\end{array}$ \\
\hline COSTE POTENCIA (euros) & 85.717 & 99.277 \\
\hline COSTE EXCESOS POTENCIA (euros) & 2.648 & 339 \\
\hline COSTE ENERGIA ACTIVA (euros) & 122.242 & 122.242 \\
\hline COSTE ENERGIA REACTIVA (euros) & 9 & 9 \\
\hline Impuesto eléctrico & 1.615 & 1.702 \\
\hline ALQUILER equipo de medida & 780 & 780 \\
\hline IVA & 44.732 & 47.113 \\
\hline TOTAL (euros) & 257.743 & 271.461 \\
\hline
\end{tabular}

Figura 1. Ejemplo real de optimización de potencias contratadas en una comunidad de regantes

\subsection{Negociación conjunta de contratos de suministro eléctrico}

Existen distintas fórmulas de agrupación de los consumidores para obtener las mejores condiciones posibles en el mercado eléctrico liberalizado: licitaciones, centrales de compras, etc. La Comunidad General de Riegos del Alto Aragón ha optado por ofrecer a sus comunidades de regantes una propuesta más flexible, donde se negocia conjuntamente desde 2010 con todas las comercializadoras de electricidad pero no existe adhesión obligatoria al resultado del proceso. Esta acción conjunta de todos los puntos de suministro ha permitido una reducción del 10\% del coste de la energía en 2015.

En la petición de ofertas se examinan modalidades no sólo con precio fijo por periodos, sino también indexadas a la evolución del mercado eléctrico mayorista.

Contrariamente a lo que sucede en otros tipos de consumidores, un contrato indexado hora a hora al mercado eléctrico mayorista de OMIE o de futuros de OMIP puede no representar oportunidad de mejora respecto a un contrato a precio fijo, dado que el periodo estival de máximo consumo eléctrico de las comunidades suele coincidir con periodos de mayor inestabilidad de precios en el mercado eléctrico. Esto se observa claramente en la Figura 2, donde se muestra un ejemplo real de una comunidad de regantes que ha optado por este tipo de contratación indexada en 2015. El coste de la energía ha resultado un $14 \%$ superior al que hubiera pagado al precio fijo resultante de la negociación conjunta de Riegos del Alto Aragón. 


\begin{tabular}{|l|r|r|r|}
\cline { 2 - 4 } & \multicolumn{3}{|c|}{ Contrado Indexado Pass-through 2015} \\
\cline { 2 - 4 } & \multicolumn{1}{c|}{$\begin{array}{c}\text { Energía } \\
\text { euros }\end{array}$} & Tarifa de acceso & \multicolumn{1}{c|}{ TOTAL } \\
\hline MAYO & 4.208 & 186 & \multicolumn{1}{c|}{ euros } \\
\hline JUNIO & 5.036 & 575 & $\mathbf{5 . 6 1 1}$ \\
\hline JULIO & 9.915 & 1.603 & $\mathbf{1 1 . 5 1 8}$ \\
\hline AGOSTO & 7.596 & 236 & $\mathbf{7 . 8 3 2}$ \\
\hline SEPTIEMBRE & 4.686 & 202 & $\mathbf{4 . 8 8 8}$ \\
\hline
\end{tabular}

34.243

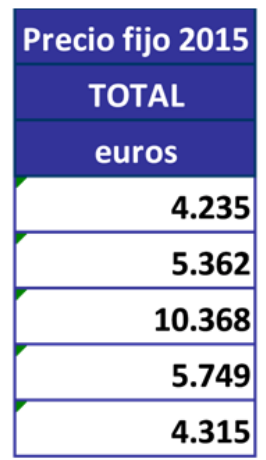

Diferencia

TOTAL

$\%$

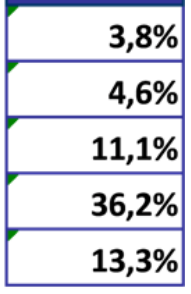

30.028

$14,0 \%$

Figura 2. Contrato indexado horario versus contrato a precio fijo en la campaña de riego de 2015

\subsection{Alternativas de suministro mediante otras fuentes de energía}

Se han realizado estudios de alternativas para analizar el ahorro que se obtendría mediante aplicación de distintas estrategias en comunidades de regantes que precisan el uso de todos los periodos tarifarios para el bombeo de agua, entre otras:

a) Reducir la potencia contratada a un valor mínimo en el periodo P1, suministrando la energía del periodo P1 con un grupo electrógeno.

b) Reducir la potencia contratada a un valor mínimo en los periodos P1 y P2, suministrando la energía de los periodos P1 y P2 con un grupo electrógeno.

c) Contratación en todos los periodos P1 a P5 de menor potencia de la demandada, aun incurriendo en excesos de potencia, manteniendo en el P6 la potencia suficiente para la instalación.

d) Contratación de suministro eléctrico durante los meses de mayor demanda y los restantes suplir la energía con grupo electrógeno (contrato de temporada)

Evaluadas en distintos escenarios y en cinco comunidades de regantes diferentes, las dos alternativas que en la mayoría de los casos resultarían más económicas son la primera y la tercera, si bien la primera precisa de inversiones mientras que la tercera necesita un seguimiento diario de los excesos de potencia registrados.

También se han realizado estudios de aplicación del nuevo Real Decreto 900/2015 de autoconsumo eléctrico, para analizar la viabilidad de disponer de instalaciones fotovoltaicas de producción de electricidad en apoyo a las estaciones de bombeo. Los resultados obtenidos no son muy satisfactorios por cuanto el plazo de amortización de las inversiones es superior a 15 años, motivado por el elevado coste de las instalaciones y la penalización al autoconsumo que introduce el citado decreto. Es preciso tener en cuenta que la rentabilidad de este tipo de instalaciones es mayor cuanto más elevado sea el porcentaje de energía producida que sea consumida, pero en las comunidades de regantes sólo se aprovecharía la producción fotovoltaica de manera más intensa en los meses de campaña de riego. Aunque en el autoconsumo tipo 2 se permite la venta de los excedentes a la red eléctrica, el precio obtenido no compensa la elevada inversión.

\subsection{Energía de reserva}

Una de las esperanzas de las comunidades de regantes de Riegos del Alto Aragón para reducir el coste del suministro eléctrico es la posible aportación de energía mediante convenios de cesión de parte de la energía reservada al Estado a la Confederación 
Hidrográfica del Ebro procedente de los saltos hidroeléctricos de Mediano y El Grado I y II, a un precio acordado de 29,72 euros/MWh.

Los estudios realizados muestran que se podría reducir un $15 \%$ el coste del suministro eléctrico a las comunidades de regantes si se aplicaran dichos convenios, cubriendo una parte de las necesidades de energía para el bombeo de agua. El ahorro está limitado por la obligación de consumir la energía en el mismo periodo horario en que se está produciendo en las centrales eléctricas, y la imposibilidad de revender la energía cedida en los periodos en que no es consumida por las comunidades.

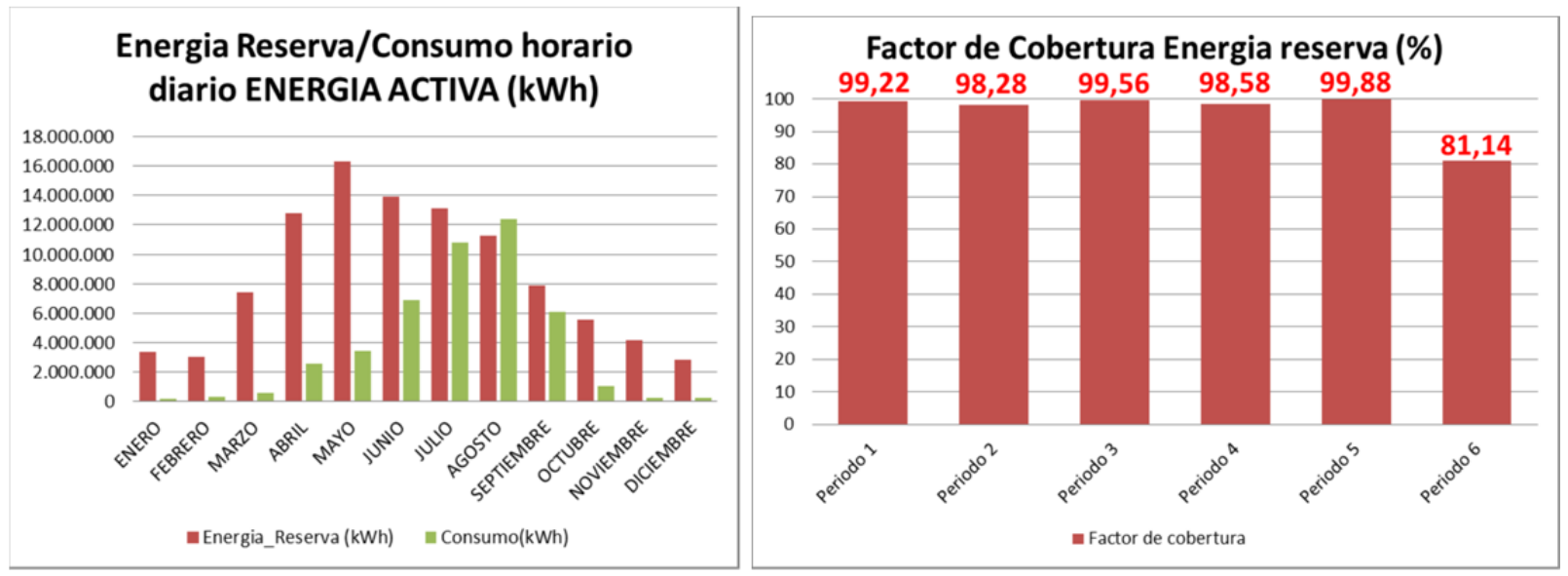

Figura 3. Cobertura que ofrecería la energía de reserva al consumo eléctrico de las comunidades de regantes

\section{Conclusiones}

Las medidas adoptadas en la gestión del suministro eléctrico de las comunidades de Riegos del Alto Aragón han supuesto un ahorro del 10\% del coste energético, lo que compensa sólo parcialmente el aumento del precio de la electricidad en los últimos años. Sin embargo hay otras fuentes de posible reducción del coste que las decisiones políticas no están favoreciendo, como el uso de energía solar de apoyo al bombeo o la cesión de la energía reservada al Estado en centrales hidroeléctricas, cuyo ahorro potencial también se ha cuantificado para prever su posible impacto en el coste energético del regadío.

\section{Agradecimientos}

A la Comunidad General de Riegos del Alto Aragón, y al Ministerio de Economía y Competitividad por su apoyo mediante el proyecto ENE2013-48517-C2-1-R.

\section{Bibliografía}

Yusta Loyo, J.M. (2013). Contratación del suministro eléctrico: oportunidades y estrategias para reducir el coste de las facturas eléctricas. Ed. Paraninfo, Madrid. 